\title{
Voix de revenants dans les Pièces pour danseurs de
}

W. B. Yeats

L'exemple de Ce que rêvent les os

Pierre Longuenesse

\section{OpenEdition}

\section{Journals}

\section{Édition électronique}

URL : http://journals.openedition.org/etudesirlandaises/4878

DOI : 10.4000/etudesirlandaises. 4878

ISSN : 2259-8863

\section{Éditeur}

Presses universitaires de Rennes

Édition imprimée

Date de publication : 15 juin 2016

Pagination : 167-181

ISBN : 978-2-7535-5091-9

ISSN : 0183-973X

\section{Référence électronique}

Pierre Longuenesse, "Voix de revenants dans les Pièces pour danseurs de W. B. Yeats », Études

irlandaises [En ligne], 41-1 | 2016, mis en ligne le 15 juin 2018, consulté le 03 mai 2019. URL : http:// journals.openedition.org/etudesirlandaises/4878; DOI : 10.4000/etudesirlandaises.4878 


\title{
Voix de revenants dans les Pièces pour danseurs de W.B. Yeats L'exemple de Ce que rêvent les os
}

\author{
Pierre LONGUENESSE \\ Université d'Artois
}

\section{Résumé}

Dans The Dreaming of the Bones, écrit sous le coup des événements de Pâques 1916 à Dublin, Yeats met en scène, jouant du modèle du Nô, un jeune insurgé en fuite, confronté à deux fantômes, à mi-chemin entre passé et présent, songe et réalité. La figure de la revenance devient alors aussi bien l'enjeu de la fable que la forme par laquelle se déploie l'écriture. D'une part, tout dans la pièce n'est que retour, puisque les événements de Pâques ravivent, à l'autre bout du temps historique, l'événement fondateur de l'oppression que fut la trahison du couple princier de Diarmuid et Devorgilla lorsque, dépossédés de leur royaume, ils firent appel aux Anglo-Normands pour le reconquérir. Et d'autre part, par une relation subtile entre le visuel et le sonore d'un côté, et les questions d'énonciation de l'autre, il revient à la voix, selon des protocoles définis, d'exprimer cette présence-absence, et ce drame d'un retour impossible. Cette forme de spectralité est redoublée par le fait que la "scène théâtrale " ne nous est donnée à voir qu'à travers les yeux - ou les songes? - d'un chœur de musiciens, sans qu'aucune didascalie ne vienne objectiver cet univers visuel et sonore en un système référentiel donné. L’action qui se déroule devant lui devient donc la projection de son univers mental, la « musique du royaume perdu » de la mémoire, surgie par la puissance de la parole. L'écriture elle-même se fait le lieu de l'affleurement du songe, par sa capacité à faire apparaitre les choses tout en manifestant leur absence. En ce sens, la pièce est une variation poétique sur la question toute shakespearienne : "Who's there? " : ce qui est en jeu est bien le retour des fantômes.

Mots clés : Yeats, Théâtre irlandais, drame poétique, dramaturgie de la spectralité, théâtre des voix, crise du drame moderne

\section{Abstract}

In The Dreaming of the Bones, written after the turmoil of the 1916 Easter Rising, Yeats, following the Noh tradition model, stages the confrontation between a young rebel fugitive and two ghosts, caught half-way between past and present, dream and reality. The figure of the past coming to haunt the present thus plays as pivotal a role in the fable as the form in which the writing unfolds. On the one hand, everything in the play is a return, as the events of the Easter Rising re-enact, from the other end of historical time, the event which gave rise to the oppression resulting from the betrayal of the royal couple, Diarmuid and Devorgilla when, dispossessed of their kingdom, they sought the assistance of the Anglo-Normans to reclaim it. On the other hand, through a subtle relationship between the visual and aural, and the new form taken by speech and enunciation, it is the role of the voice, according to defined protocols, to express this presence-absence, and the drama of an impossible return. The spectral projection is furthermore intensified by the fact that the "theatrical representation" is only conveyed to us through the eyes - or possibly dreams - of a group of musicians, without any stage direc- 
tion which might situate this visual and aural universe in a given system of reference. The action that unfolds before the spectator thus becomes a projection of his imaginary landscape, the "music of the lost kingdom" of memory, brought forth by the power of utterance. In this manner the writing itself becomes the place where dreams can emerge through its capacity to conjure up things that are simultaneously shown to be absent. Thus, the play is a poetic variation on the question "Who's there?".

Keyword: Yeats, Irish theatre, poetic drama, dramaturgy of spectrality, theatre of voices, crisis of modern drama

Dans The Dreaming of the Bones, pièce écrite sous le coup des événements de Pâques 1916 à Dublin, trois musiciens-narrateurs sont les spectateurs autant que les ordonnateurs d'une scène unique dans laquelle un jeune insurgé en fuite, perdu dans une lande improbable au fin fond du Comté de Clare, est confronté à deux figures fantomatiques venues du passé, perdues dans les méandres de leur mauvaise conscience : ces deux revenants ne sont autres en effet que le couple de Diarmuid et de son amante Devorgilla qui, au XIII ${ }^{\mathrm{e}}$ siècle, firent appel aux Anglo-Normands pour reconquérir leurs terres suite à leur bannissement, et qui, depuis, ne cessent d'errer en quête du pardon qui rachètera leur faute. Le motif du retour fonde donc dans cette pièce la construction même de la fable, puisque les événements de Pâques ravivent, à l'autre bout du temps historique, l'événement fondateur de l'oppression anglaise que fut cette trahison, sept siècles avant 1916. Qui plus est, et comme l'on sait, Yeats s'inspire pour cette pièce comme pour d'autres du modèle du Nô japonais, en opposant un personnage venu du monde réel, le waki du Nô - ici, le jeune homme - à un esprit ou un revenant, le shite ${ }^{1}$ - ici, les deux amants.

Dès lors, lorsque les deux personnages fantomatiques posent la question : «Pouvons-nous être pardonnés? "Ils en posent en réalité une autre, aussi simple qu’efficace dans sa théâtralité ${ }^{2}$ : pouvons-nous revenir? Dans leur désir de retour, ils sont

1. Dans le Nô, le waki est un étranger qui vient visiter un lieu retiré et hanté, et est confronté à un revenant, à une vision spectrale, le shite, personnage dans l'entre-deux de la vie et de la mort. On peut suggérer une analogie entre la figure masquée du shite et l'eidolon grec, cette figure venue de ces espaces autres que sont similairement le monde des morts ou celui des dieux. On ne reviendra pas sur les circonstances de la découverte du Nô par Yeats à travers ses liens avec Ezra Pound, ni sur le contexte de cette découverte, marqué par un fort regain d'intérêt pour le Japon en général en ce début du $\mathrm{xx}^{\mathrm{e}}$ siècle, intérêt ravivé par la découverte des manuscrits de Zeami en 1910, suivi très rapidement par leur publication. Ernest Fenollosa (1853-1908), historien d'art et philosophe, vécut une quinzaine d'années au Japon dans les années 1880 et 1890. Professeur à l'Université et Directeur de l'Académie des Beaux-Arts de Tokyo, puis conservateur au département d'art oriental au musée des Beaux-Arts de Boston, il meurt prématurément, laissant en friche une œuvre considérable. Sa veuve demande à Ezra Pound de publier les carnets de son mari. Pound publie donc en 1914 le Nô Nishikigi-qui inspira The Dreaming of the Bones -, puis en 1917, l'essai Nô, or Accomplishment, une étude sur la scène classique japonaise. L'article de Yeats Certain Noble Plays of Japan constitua l'introduction de ce dernier ouvrage.

2. On envisage ici le terme théâtralité à partir de son étymologie grecque : est " théâtral " ce qui littéralement se donne à voir, et induit l'opsis, la vision, qui elle-même est le fondement de toute représentation, mettant face à face un regardant et un regardé. C'est en ce sens que la question posée par les deux fantômes est proprement théâtrale : peuvent-ils « revenir » ou, en d'autres termes, ré-apparaître pour être vus. 
à la fois déjà là, et pas encore complètement « là ", balançant entre absence et surcroît d'être. Cette question fait d'eux, plus que des actants, des " étants ", ou en quelque sorte des « aspirants à être ». Comme dit Jacques Derrida, « il y a du disparu dans l'apparition même comme réapparition du disparu ${ }^{3}$ ». La spectralité, comme le laisse penser son titre, est au centre de la pièce The Dreaming of the Bones.

Pour le chercheur, la question n'en reste pas moins de savoir comment s'exprime cette spectralité dramaturgiquement, et s'il y a chez Yeats une dramaturgie spécifique de la revenance. Comment Yeats s'y prend-il pour ne pas représenter un spectre de façon totalement incarnée, procédé qui serait contradictoire dans les termes? Comment trouve t-il les formes adéquates, aussi bien textuelles que scéniques $^{4}$, de la spectralité ${ }^{5}$ ? On examinera donc deux aspects majeurs de l'écriture dramatique de cette pièce - le rapport entre le visuel et le sonore d'une part, et les questions d'énonciation d'autre part - en tentant de voir comment c'est bien à la voix, selon des protocoles très définis, qu'il revient d'exprimer cette présenceabsence, et ce drame d'un retour impossible.

\section{圈 Du visuel et du sonore, de l'invisible et du dicible}

\section{Du sonore au lieu du visuel}

Le premier procédé visant à la mise en forme de cette idée de "revenance " dans l'écriture est en effet la dissociation entre les deux "media " visuel et sonore. La fiction dramatique se déroule la nuit. Dans le texte cette indication est distillée

3. Jacques Derrida, Spectres de Marx, Paris, Galilée, 1993, p. 25.

4. L'objet de cette étude est l'analyse du texte, tout en gardant à l'esprit l'horizon que constitue la scène. Du reste, au delà de la structuration de la parole et des regards, et de la question des acousmates, qui vont être traitées ici, le texte inscrit également dans ses didascalies des directions soumises aux praticiens - notamment les masques, et les jeux d'apparition/disparition des personnages grâce à l'étoffe dépliée et repliée par les musiciens. Une étude reste à faire sur la création de la pièce au Peacock, la petite salle de l'Abbey Theatre de Dublin, le 6 décembre 1931, soit quatorze ans après son écriture, et dix après son édition. On sait qu'une musique fut rapidement commandée au compositeur Walter Rummel, mais qu’elle ne fut jamais jouée. A la création, Udolphus Wright assurait la mise en scène, et ce fut John Larchet, directeur musical de l'Abbey, qui créa la musique de scène, pour un chanteur, une flûte, une percussion et un "zither ", ou petite harpe, proche du psaltérion.

5. Dans une lettre à Lady Gregory, Yeats confie : "I believe I have at last found a dramatic form that suits me " (lettre à Lady Gregory du 26 mars 1916, in Allan Wade (dir.), Letters, London, Rupert Hart-Davis, 1954, p. 609). Comme on l'a dit plus haut, Yeats s'inspire d'un canevas de Nô existant pour écrire The Dreaming of the Bones: il en respecte donc la structuration des personnages et de la trame. Mais son entreprise n'est pas tant archéologique que poétique. Les codes du Nô n'ont été pour Yeats que les outils adéquats lui permettant de rompre avec les modèles dramatiques dominants. Il ne s'agit donc pas tant de prendre à la lettre ces codes que de s'intéresser plutôt au champ dramaturgique nouveau qu'ils lui ont permis de dessiner : celui d'une subtile architecture de paroles, enchâssées, en contrepoint, en alternance, déléguées, d'une partition de musique avec ou sans musique, partition d'une cérémonie ritualisée au service d'une initiation métaphysique. Les Plays for Dancers ont pour objet le retour spectral du mort, dans un instant d'épiphanie éphémère située au climax de la pièce. L'écriture de The Dreaming of the Bones sous l'influence du Nô permet ainsi une subtile inscription de l'actualité politique (la révolte de Pâques 1916 à Dublin) dans une mythologie nationale héroïque. 
de façon parfois directe, et souvent indirecte, soit dans les répliques didascaliques et donc narratives des musiciens, soit de façon plus lyrique dans leurs parties chantées, soit enfin dans les répliques des protagonistes eux-mêmes : "What rogue is night wandering ${ }^{6}$ ? "; "And many a night it seems $[. . .]^{7}$ "; " The hour before dawn and the moon covered $u p^{8}$ "; "Why have you come creeping through the dark? ?"; " [I] would break my neck/If I were stumbling there alone in the dark ${ }^{10}$ "; " He has been wandering on the road all night ${ }^{11}$. "Plus loin cette ambiance nocturne nous est rappelée par la mention du "night wind ", jusqu'à la fin où le motif est repris par les musiciens : "I have heard in the night air ${ }^{12}$." Quant au jeune homme, il marche sur les routes tenant à la main une lanterne, dont la faible lueur ne fait qu'accuser la nuit. Et un peu plus loin, les oiseaux de nuit (le hibou, mentionné trois fois) ne cèdent pas la place au coq, qui ne chante pas encore. En un mot, toute la pièce repose sur l'attente en même temps que la crainte du jour. Dans ce contexte, l'invisibilité est constamment rappelée, et cette invisibilité a pour corollaire une valorisation du medium sonore. Symptomatiquement, c'est sur ce mode que s'ouvre l'action dramatique. C'est par sa voix que se manifeste d'abord le jeune homme, après que le bruit de ses pas a précédé son arrivée dans le récit des musiciens :

First musician : [...] I hear a footfall -

A young man with a lantern comes this way.

[...] He stumbles wearily, and stumbling prays.

A Young Man enters, praying in Irish ${ }^{13}$.

De la même façon c'est par une question que s'ouvre la première réplique chantée des musiciens, avant même l'entrée du jeune homme, et cette question porte sur ce dont on pressent la présence sans rien en voir : " Did not a shadow pass? [...] Who can have trod in the dark? » Ce questionnement est redoublé par le jeune homme qui interroge à son tour des interlocuteurs qu'il ne voit pas :

Young man: Who is there? I cannot see what you are like! Come to the light ${ }^{14}$.

En guise de réponse la jeune fille souffle la bougie, le plongeant dans le noir. La lanterne éteinte, on ne perçoit plus des personnages que leurs voix. Plus loin il

\footnotetext{
6. William B. Yeats, Collected Plays, Londres, Macmillan, [1934], 1982, p. 433.

7. Ibid., p. 434.

8. Ibid.

9. Ibid., p. 435.

10. Ibid.

11. Ibid., p. 436.

12. Ibid., p. 445.

13. Ibid., p. 434.

14. Ibid., p. 435.
} 
est aussi question du bruit d'un cheval que l'on entend sans voir : "What is that sound? - An old horse gone astray $[\ldots]^{15}$."

Cette focalisation sur le signifiant sonore, au détriment de sa source, renvoie aux théories acousmatiques développées aussi bien par l'antiquité pré-socratique que par les musicologues contemporains. Le concept d'acousmate, dans son sens pythagoricien originel, désigne en effet un son que l'on entend sans en déceler les causes :

Acousmatique n.m. (du grec Akousmatikos, habitué à écouter) Philos. Nom donné aux disciples de Pythagore qui, pendant cinq années, écoutaient ses leçons cachés derrière un rideau, sans le voir, et en observant le silence le plus rigoureux. Adj. Mus. Se dit d'une situation d'écoute où, pour l'auditeur, la source sonore est invisible; se dit d'une musique élaborée pour cette situation ${ }^{16}$.

Cette distorsion entre vision et audition crée un système d'interprétation des signes qui indique une présence-absence. Ce qui est désigné par un signe sonore est suffisamment présent pour qu'il y ait signe et en même temps suffisamment absent pour qu'il y ait questionnement sur le sens de ce signe. Roland Barthes, dans L'Obvie et l'obtus, explique comment cette posture de guet s'accompagne d'une volonté de déchiffrement. Lorsque l'audition, phénomène physiologique, se mue en écoute, acte psychologique, le personnage est entièrement tendu vers la captation et l'interprétation de signes extérieurs ou intérieurs : "L'écoute ne peut se définir que par son objet, ou si l'on préfère, sa visée [...]. L'être vivant tend son audition vers des indices [...]. Ce qu'on essaye de capter par l'oreille ce sont des signes ${ }^{17}$. " Cette focalisation sur l'audition est donc à l'origine d'une dynamique dramatique fondée sur la tension entre le " monde réel " des personnages - en l'occurrence ici le jeune fugitif - et des figures spectrales, hommes ou bêtes, qui ne cessent de le hanter et le menacer. Une sorte de double-jeu - ou de jeu de double? - s'instaure entre le personnage et sa voix, et la fable devient comme " hantée " par son ombre, par la présence/absence, en creux, d'une altérité non identifiée. La voix coupée du corps se fait étrange. Freud dans son article sur "L'Inquiétante étrangeté " précise que c'est " tout ce qui se rattache [...] aux cadavres, à la réapparition des morts, aux spectres et aux revenants ${ }^{18}$ ", qui apparaît de façon récurrente le plus "étrangement inquiétant ». Il articule cette remarque sur les phénomènes de « redoublement du moi, scission du moi, substi-

15. Ibid., p. 436.

16. "Acousmatique ", Grand Larousse Universel, 1995.

17. Roland Barthes, L'obvie et l'obvus, Paris, Seuil, 1982, p. 215.

18. Sigmund Freud, "L'inquétante étrangeté " (Das Unheimlich), in Essais de Psychanalyse appliquée, Nrf Gallimard, coll. «Idées », 1976 [1933], p. 194, traduit de l'allemand par Marie Bonaparte et $\mathrm{M}^{\mathrm{me}}$ E. Marty. 
tution du moi ", où " le signe algébrique du double [...] devient un étrangement inquiétant signe avant-coureur de la mort. " Bref, la frontière se brouille entre vivants et morts ${ }^{19}$.

\section{Bruits silencieux, spectres au milieu des spectres}

Il y a donc un premier niveau, simple, de construction dramaturgique de la revenance. Celle-ci se manifeste vocalement par le jeu des acousmates : des bruits entendus dont on sait la source existante, avérée par une didascalie ou une indication du discours, mais qu'on ne voit pas. La voix qui prie. Le bruit du cheval. Les voix des deux fantômes.

Cela dit, à côté de ce paysage sonore, une deuxième trame est bientôt discrètement perceptible, autrement plus importante et plus dense. En effet le discours des personnages - en particulier des deux fantômes - est littéralement peuplé d'un grand nombre de présences évoquées au fil de leur cheminement dans les lieux du passé. Et, comme par hasard, toutes leurs manifestations sont sonores : les moines, les animaux, les objets, les cloches, tout bruisse. Ce phénomène est amplifié par un jeu d'écho entre les répliques des protagonistes et celles des musiciens, comme si la parole des musiciens jouait le rôle d'une caisse de résonance. Au début de la pièce, par exemple, il est question des morts qui hantent ces lieux désolés. Le jeune homme les rassemble dans une même image autant visuelle que sonore : "Let them dream... and fill waste mountains with the invisible tumult of the fantastic conscience ${ }^{20}$. " Juste après, l'étranger introduit sans transition le motif du cri $\mathrm{du}$ coq : "before the cocks... shake their wings and cry ${ }^{21}$ ». Puis c'est le musicien qui revient, sur le mode sonore, aux personnages du passé "The narrow lane where mourners for five centuries have carried/ Noble or Peasant to his burial ${ }^{22} "$. À cette image il entremêle d'autres fantômes du présent, le "drinking cattle ", le troupeau qui traverse la montagne; il reprend ensuite au jeune homme le motif du coq dans son chant en y introduisant une variante, puisque l'expression "shake their wings" devient "clap the wing", et "cry " devient "crow». Plus loin, dans leur chant, les musiciens élargissent encore cet univers de rumeurs. Au lieu du coq c'est la chouette nocturne qui "cry out above their heads", puis qui à la page suivante bat des ailes mais différemment : "beats with a vague wing". Puis vient le cri de l'oiseau à tête de chat : "The Cat-headed bird is crying out ${ }^{23}$. " Encore plus loin

19. Georges Didi-Huberman développe longuement ces figures de la spectralité au théâtre, et les manifestations d'une dissolution du sujet, en soulignant l'étymologie commune des termes « spectacle » et "spectral »; voir Génie du non-lieu, Paris, Minuit, 2001, p. 121.

20. William B. Yeats, Collected Plays, op. cit., p. 436-437.

21. Ibid., p. 437.

22. Ibid.

23. Ibid., p. 438. 
d'autres " images acoustiques " renforcent l'expressionisme de ce paysage sonore, en entremêlant sans cesse passé et présent dans une même représentation mentale; à son tour l'étranger évoque les fantômes du passé, comme s'il reprenait au vol la parole du musicien, en la précisant ou la détournant :

In the old days we should have heard a bell Calling the monks before day broke to pray ${ }^{24}$.

Il insère dans le tableau du passé le motif du coq, mais cette fois-ci avec le terme "crow": "The crowing of its cocks ${ }^{25}$ ". À la fin de la pièce enfin il y aura d'autres cris encore dans le chant final des musiciens : " the curlew cry before dawn », avant la reprise du cri du coq.

Bref, il s'agit d'un vrai concert. Un paysage sonore se déploie dans la parole dialoguée, et se réverbère, en contrepoint, dans le discours lyrique des musiciens. Les uns et les autres se passent le relais, et ces paroles croisées organisent une géographie imaginaire, un cymbalum mundi des oiseaux, des objets, des éléments, et des êtres humains, allant et venant entre passé et présent. Dans le même temps pourtant, tout bruisse silencieusement. Ce concert est un concert muet, l'évocation sonore est purement textuelle. On se trouve face à une sorte de second degré de présence-absence : des bruits silencieux entourent d'autres bruits, eux-mêmes signes d'une présence absente.

Yeats met donc en place d'abord une structure acousmatique de surface - les voix des personnages. Organisées choralement, ces voix sont à leur tour le véhicule d'autres figures acousmatiques, plus purement mentales, représentantes d'un univers archaïque, mythologique, voire surnaturel. Dans les mots affleurent ainsi une mémoire, un lointain intérieur, un imaginaire sonore, d'appels, de cris, de martèlement, de heurts, de musique, enfoui dans l'oreille interne, qui se manifeste par rémanence par le biais des acousmates - le paysage qui se construit est un "paysage intérieur ", un univers éso- plutôt qu'exo-térique, un théâtre mental construit par les pouvoirs du verbe.

\section{La revenance, ou le passage du sonore au visuel}

Mais reprenons d'un peu plus près l'un de ces acousmates : celui des oiseaux; ils sont mentionnés deux fois par les musiciens au début de la pièce : "Birds cry, they cry their loneliness", puis plus loin : "Once more the birds cry in their loneliness $^{26}$. "Ce motif révèle une sorte de double collage : entre le mental et le représenté d'une part, et entre le sonore et le visuel d'autre part. La mention des

24. Ibid., p. 439.

25. Ibid.

26. Ibid., p. 434. 
oiseaux précède en effet immédiatement l'apparition des deux fantômes. Autrement dit, les musiciens se les représentent d'abord mentalement, sous une forme poétique et fantastique, avant qu'ils ne surgissent devant leurs yeux et devant les nôtres. De plus cette représentation mentale est en fait une hallucination sonore. Par cette circulation entre la représentation mentale acousmatique et l'actualité de la scène, une parole hallucinatoire fait surgir une présence. Un autre exemple vient confirmer cette technique de collage lorsqu'à la mention des pas du jeune homme succède son arrivée acousmatique par le bruit de la prière. Cette succession semble anodine, mais le décalage entre ces deux acousmates, l'un mental (le bruit des pas), et l'autre réel (le bruit de la prière), est poétiquement productif. Rien n'empêchait Yeats de créer une didascalie mentionnant le bruit des pas. Et quand bien même il en aurait fait l'économie simplement à cause de leur mention dans le discours des musiciens, le résultat est le même. De fait, les musiciens ne commentent pas une action qui leur est extérieure : cette action est d'abord mentale. Le personnage n'entre pas de lui-même, c'est le discours des musiciens qui le fait surgir. Cette impression est d'ailleurs confirmée par le mode sur lequel, à la fin de la pièce, le personnage sort : en fait, il ne sort pas, il disparaît, happé par le tissu déroulé des musiciens : "While it is unfolded, the Young Man leaves the stage $^{27}$. "L'objectivité de sa présence est donc relative, et strictement dépendante de la parole.

La pièce met donc en scène, de par la relation entre la parole, le son, et l'image, le processus de l'incarnation d'un songe. Fidèle à une technique qu'il développe ailleurs, Yeats enchâsse les uns dans les autres plusieurs niveaux de rêverie. Celle des musiciens d'abord, qui font surgir les personnages de leur imagination : leur verbe se fait chair, et leur texte lyrique est en quelque sorte le lieu d'un pré-conscient : la scène, quant à elle, cristallise le songe. Mais aussi la rêverie des personnages eux-mêmes, puisque les acousmates expriment également ces phénomènes d'ordre hallucinatoire, frappés d'irréalité, que sont les souvenirs auditifs ou visuels. La " musique d'un royaume disparu ${ }^{28}$ " se déploie donc d'abord dans ces signes de l'absence. Dans ce contexte, la dernière réplique chantée des musiciens opère une synthèse de cet univers de mémoire fragmentaire. Loin en effet de constituer une clôture lyrique triomphante, un retour à la fermeté d'un « je » parlant et chantant, cette réplique ne fait que prolonger une esthétique du tâtonnement et du fragmentaire. La parole des musiciens s'y déroule en effet en vers brefs, et les constantes reprises de termes, ou variations, et les jeux d'homophonie parfois très virtuoses, sont comme autant d'approches par touches successives des fragments d'une mémoire ou d'un souvenir, fragments que l'on

27. Ibid., p. 444.

28. Ibid. Ce sont les mots des musiciens dans leur chant final : "At the grey round of the hill/ Music of a lost kingdom/ Runs, runs and is suddenly still." 
rassemble par des tentatives sans cesse incomplètes de nomination. Plus ces différentes touches sont redites, plus elles sont frappées d'absence, dans leur surréalité même. Le chant qui se construit là freine toute tentative d'épanchement lyrique, puisque les répétitions brisent à chaque fois toute possibilité de déploiement vocal. En fait, l'écriture ramène sans cesse la présence des musiciens à leur stricte voix, qui dit la simple tentative d'être à soi et d'être dans le monde, comme s'ils participaient eux-mêmes, par compassion ou par confusion des frontières entre sujet et objet de la vision, à ce drame de la revendication d'être que constitue The Dreaming of the Bones.

\section{La question de l'énonciation : l'être et la voix}

Cette dernière remarque sur la voix des musiciens nous met, au demeurant, sur le chemin d'un autre procédé de mise en scène de la revenance. Au-delà en effet du phénomène des acousmates, qui reste du domaine d'un questionnement sur la représentation, on se rend compte que c'est la voix en elle-même qui exprime la spectralité. L'idée n'est évidemment pas nouvelle, mais on peut pourtant examiner avec intérêt le processus par lequel le poète dramatique Yeats fait de ses deux fantômes de pures voix - et bien sûr il faudra analyser ce concept -, à mi-chemin entre une figure absente et une présence incarnée dans l'actualité d'un corps. Au cœur de ce processus, il y a l'organisation énonciative du texte, que l'on examinera sous deux angles particuliers : le traitement des questions de personne et la rythmique du discours.

\section{La question de la personne}

La question fondatrice de la pièce : "who's there? ", n'est en effet pas seulement de l'ordre d'une situation physique incertaine, où le son permet de représenter ce stade intermédiaire entre visibilité et invisibilité. On remarque qu’à cette question répond une autre question, et que les deux fantômes ne font, au fil de la pièce, qu'un rare usage de la première personne. Et cette quasi absence de je est d'autant plus remarquable que durant toute la scène, par contraste, le jeune homme ne cesse de s'affirmer dans un discours souvent agité, interjectif, interrogatif, exclamatif - on rencontre pour ce qui le concerne vingt-trois occurrences de première personne dans son discours, si l'on y inclut toutes ses formes, y compris possessif ("my») ou pronom objet (" me ») ou pluriel ("we»). Celui qui répond à une question par une autre question - en l'occurrence l'étranger - reste donc non identifié. Dans toute la pièce, il n'emploie la première personne que sept fois, dont deux seulement à la première personne du singulier : dans « we know 
the pathways that the sheep tread out ${ }^{29}$ ", le " nous " est une première personne $\mathrm{du}$ pluriel qui inclut implicitement la jeune fille, prenant ainsi acte du fait qu'ils ont été perçus, sinon vus, tous les deux, comme en témoigne la phrase du jeune homme quelques répliques auparavant : "I saw a pair of heads against the sky. " Ensuite, on rencontre deux occurrences inattendues, et assez spectaculaires, d'un " je » à la première personne du singulier : "I will put you safe,/No living man shall set his eyes upon you;/I will not answer for the dead ${ }^{30}$. "C'est la seule fois où l'étranger coupe la parole au jeune homme, et ce pour introduire le motif de la présence des morts - comme s'il tentait d'emblée le tout pour le tout, tendant au jeune homme une perche pour se faire reconnaître. Cette tentative ayant échoué, on ne rencontre par la suite plus un seul je dans sa bouche; les trois derniers "nous " sont des "nous" inclusifs et neutres : "We are soon among the stones ${ }^{31}$ ", puis "We're almost at the summit and can rest [...] " et "In the old days we should have heard a bell' ${ }^{32}$. La parole de l'étranger s'efface derrière les noms de lieux et les visions, comme une parole narratrice.

Celle de la jeune fille, de son côté, est encore plus transparente. Silencieuse durant toute la première partie de la pièce, elle fait irruption dans le discours de son compagnon en cours de vers, prenant le relais de l'étranger comme si elle ne faisait que se glisser dans sa peau et dans son discours, comme son ombre. Ensuite elle parle longuement à la troisième personne, évoquant sur le mode épique et narratif les personnages qu'ils sont sans pouvoir l'être : comme si l'instance énonciatrice de ce discours constituait bien le fantôme égaré au présent des figures du passé. Pour tenter de faire se superposer enfin ces doubles, une seule première personne survient enfin "Yes, yes, I spokelOf that most miserable, most accursed pair $[\ldots]^{33}$ ». Mais ce « je », en surgissant au moment même où le jeune homme prononce leurs noms, Diarmuid et Devorgilla, ne fait en réalité que creuser l'écart entre les deux termes du double, qui restent séparés. Du reste, il faut remarquer que la dernière occurrence d'une première personne est d'une grande tension dramatique. Le vers "Seven hundred years our lips have never met " prononcé finalement par la jeune fille ${ }^{34}$ est une reprise-variation explicite du vers qu'elle a prononcé peu de temps auparavant : "Though eyes can meet, their lips can never meet ${ }^{35}$." D'un seul coup, la troisième personne devient première : il s'opère enfin une soudaine superposition entre sujet et objet du récit - et ce, au moment même de leur disparition. Le moment même du retour effectif, c'est-à-dire de l'incarna-

29. Ibid., p. 435.

30. Ibid., p. 436.

31. Ibid., p. 437.

32. Ibid., p. 438-439.

33. Ibid., p. 442.

34. Ibid., p. 443.

35. Ibid., p. 441. 
tion, constitue une fugitive épiphanie, qui coïncide avec l'effacement des personnages - nous y revenons plus loin.

\section{Le rythme du discours}

Si les fantômes ne prennent donc en quelque sorte jamais leur place dans le procès de la parole, l'organisation énonciative de cette parole se caractérise également par un rythme particulier, en l'occurrence celui d'une " pensée parlée " se déroulant dans un temps constamment suspendu, comme en apnée respiratoire. Dans ce trio vocal que constitue l'échange entre les trois personnages, la première voix, celle du jeune homme, est très incarnée, sur un rythme vivace. Le rythme des deux autres, au contraire, est ralenti par les suspensions dues aux nombreuses propositions incises à l'intérieur des vers. Les répliques de l'étranger sont quasi systématiquement ralenties par des circonstancielles ou des compléments qui séparent le sujet de son verbe, ou l'auxiliaire de son participe passé. Structuré par de puissantes armatures syntaxiques, le discours est scandé par des anaphores. Le souffle de la réplique est, de fait, suspendu : les reprises d'inspiration, ou les expirations, sont sans cesse retenues ou différées. Les répliques de la jeune fille, quant à elles, amplifient largement ce phénomène, relançant le contraste avec la voix du jeune homme. On peut citer le cas limite d'une réplique de quatorze vers constituée d'une seule phrase ${ }^{36}$. Dans celle-ci, la construction syntaxique est source d'une grande tension entre clôture et relance, et oblige à une lenteur de la lecture. Expérimentant la limite jusqu’à laquelle il peut différer la résolution de sa phrase, Yeats ajoute vers après vers un degré de tension dans l'esprit et l'oreille $\mathrm{du}$ lecteur/spectateur. Du premier au treizième vers, trois occurrences successives de "Although" (la première et la troisième au début des vers 1 et 8 , la seconde au milieu du vers 5) maintiennent fermement la réplique dans sa logique discursive, et encadrent trois sous-ensembles eux-mêmes armaturés par d'autres verbes, non sans introduire au demeurant un troisième étage de subordination (une relative des vers 2 à 5 , une autre au vers 7, se faisant évidemment écho par la reprise de "who»). Ce sont ces subordonnées incises, ou des verbes au participe présent, qui permettent de différer encore un peu plus le verbe principal et de donner toute son amplitude à cette vaste architecture verbale.

On le voit donc, la parole est elle aussi le lieu d'une tentative de retour, et le théâtre de la revenance se joue dans la prise ou la non-prise de parole des personnages : si, comme dit Jakobson, il y a une relation existentielle entre le déictique « je » et l'instance de l'énonciation, (puisque « je » désigne celui qui dit « je »),

36. Il s'agit de la réplique de la jeune fille : "Although they have no blood, or living nerves, [...] And drive them apart." C.P., p. 441. 
alors l'absence de « je » pose la question de l'impersonnalité. Deux corps qui ne disent pas « je » se retirent dans deux voix qui elles-mêmes retiennent leur souffle : le mode de déploiement de cette double voix désigne bien cette instance qui fait retour, et qui se tient dans une posture de vouloir-dire, et en même temps parlant depuis le lieu où elle ne peut rien dire.

\section{La parole épiphanique, de la résurgence à la résurrection}

On aborde ici le point d'arrivée de notre raisonnement. L'horizon dramatique de la pièce est en effet cet épisode épiphanique final que met en scène la dernière réplique du jeune homme, où se résout la tension entre être et non-être, entre le désir d'arrachement au passé et l'impossibilité d'une incarnation au présent. Dans cette réplique en effet, le jeune homme semble décrire une action : celle des deux fantômes qui s'éloignent, dansant, et disparaissent au lointain. Rapidement pourtant, ce mouvement se déplace du visuel au sonore, se concentrant dans la dynamique rythmique des mots que vient appuyer la réapparition des instruments. Le temps des verbes (au present perfect) donne la sensation d'un temps incertain, non linéaire, et favorise cette perception plus sensible que narrative d'une masse d'actions prises dans leur ensemble. La parataxe, les accumulations de verbes, les enjambements ou contre-rejets, créent une tension entre étirement et brisure, continuité et discontinuité, élan et chute, inspiration et affaissement. Les répétitions, par une dialectique subtile entre le même et le différent, sont à l'origine d'un effet de spirale : le mouvement est à la fois circulaire et ascensionnel, mouvement d'éloignement et de fuite, jusqu'à la disparition des personnages engloutis ou aspirés par la brume.

Cette danse des mots est donc la cristallisation même du revenant-partant. C'est le départ de ce qui n'est pas arrivé à revenir - et du coup c'est l'arrivée de ce qui s'en va. Si la chose était complètement là, elle n'apparaitraît plus. C'est donc bien dans la partance que se joue le stade exaspéré de la revenance. C'est dans la disparition que se joue l'apparition. Pour Yeats, le ballet des mots joue justement à étirer cet instant de l'entre-deux. L'action ne se situe ni "dans le monde ", ni même dans l'esprit, de celui qui parle, mais bien dans sa voix. Du reste, rien ne nous dit dans le texte que ces deux fantômes dansent effectivement, ou au contraire ne dansent pas : bien sûr, le terme " dance " est repris abondamment par le personnage du jeune homme, aussi bien dans la dernière réplique dont nous parlons, que dans l'une des répliques précédentes. Mais aucune didascalie ne le spécifie, et ne confirme pour nous l'effectivité de cette danse. L'hypothèse a minima que l'on puisse faire est que le jeune homme la voit; et à coup sûr, elle est dans sa parole. Il n'y a donc ni danse, ni absence de danse, mais peut-être le troisième terme d'une danse-dans-les-mots. Du reste, si la danse d'effacement et 
de disparition des fantômes est ainsi, comme nous le suggérons, un jeu verbal, la triple répétition (à certains égards inutile en soi) du terme "dance " lui-même, et l'effet rythmique qu'elle induit, en constitue précisément une ultime preuve tout comme le quasi-anagramme constitué par les lettres, autant que les sons, du mot " hands ». L'hallucination par la parole se substitue à l'envoûtement par la danse. C'est en cela que nous pouvons dire, concernant Yeats, que la parole est épiphanique, et sur cette question de la présence de la danse, au final, la décision n'appartient qu'au metteur en scène. Et si l'on peut se permettre d'énoncer ici un point de vue, il ne serait probablement pas judicieux de créer une redondance inutile en "illustrant " la parole du jeune homme par une danse des deux fantômes : le metteur en scène est mis au défi de trouver un moyen tel que ce soit la parole qui fasse voir cette danse.

Dans ces nouvelles règles du jeu, que devient la revenance? L'écriture, en effet, en ne s'articulant ni à un référent-dans-le-monde, ni à un référent-sur-scène (ou du moins pas physiquement manifesté en dehors de la voix), construit un univers de mouvement et de vision, par auto-référence, en s'appuyant sur la voix et le souffle. Dans une perspective théâtrale, cette "voix de l'écriture ", parce que sa nature est autant, voire plus, pneumatologique que grammatologique ${ }^{37}$, pose donc en termes nouveaux la question de la représentation. Le texte est foncièrement non-référentiel, parce qu'il devient auto-référentiel, en ne renvoyant plus quà lui-même : le signifiant se rabat sur le signifié, et la rythmique des mots double l'image poétique des personnages dansant. Dans le ballet des mots il n'y a plus alors de revenance, il n'y a plus que de la performance : la musique de la parole, et son incarnation par la voix du comédien, soutenue par la rythmique des instruments, se déroule dans "l'ici et maintenant " de sa profération, sans renvoyer à quelque représentation que ce soit ${ }^{38}$. Il ne s'agit plus d'évoquer de façon indicielle un univers invisible, mais d'incarner un monde en quelque sorte " parallèle ", double, et peut-être métaphore, du monde réel. La parole, en tant que souffle, rythme et mouvement, est en soi incarnation. Celle-ci s'opère de façon magique, épiphanique : la frontière entre celui qui regarde et ce qu'il regarde s'est estompée, le protagoniste parlant s'efface, se dissout, par sa propre voix, dans la vision qui l'habite, donnant à cette vision une dimension apocalyptique, de révélation. Dans

37. Jacques Derrida, De la grammatologie, Paris, Minuit, 1967, p. 29.

38. À une exception près - et c'est encore un paradoxe de cette pièce, et non des moindres : si aucune didascalie ne signale une danse, alors que l'horizon même de la pièce semble être la danse des fantômes, à trois reprises il est question d'une marche: "They go round the stage once. " Ces marches semblent représenter quelque chose : comme nous le suggèrent les commentaires des musiciens, chacune d'entre elles correspond, symboliquement, à une étape du chemin vers l'abbaye. Mais à certains égards, elles sont aussi une belle preuve de la porosité, dans la dramaturgie de Yeats, entre représentation et performance : tendant vers l'auto-référentialité, elles ne sont aussi signes que d'elles-mêmes, comme si les «stations » du chemin vers la révélation/disparition des fantômes n'étaient, dans l'ici et maintenant du spectacle, qu'à la fois du souffle et du mouvement, de la voix et du corps. 
ce contexte, il n'est pas anodin que le propos de cette pièce soit la catastrophe de Pâques 1916, et la confrontation à l'indicible de la mort.

\section{圈 Conclusion}

L'écriture dramatique de Yeats fait de lui un musicien du seuil, des limites, du passage. Les acousmates organisent un orchestre sonore d'objets et d'êtres dont une partie est dans l'ici et l'autre dans l'ailleurs. On se situe dans la catégorie psychologique du double, et dans une tentative de mise en scène de la mort, ou du mort, au théâtre :

Accueillir [...] mais tout en appréhendant [...] un étranger qui se trouve déjà au-dedans, plus intime à soi que soi-même, la proximité absolue d'un étranger dont la puissance est singulière et anonyme, une puissance innommable et neutre ${ }^{39}$.

Pour mémoire, ce qui definit l'eidolon archaïque, c'est sa valeur de présence dans le visible d'une puissance de l'ordre de l'invisible, qu'elle soit des morts ou des dieux. Dans cette configuration dramatique, la voix est le lieu par excellence de la représentation du " déjà là » et du " pas encore là », l'inscription de l'ailleurs dans l'ici. On peut rappeler ces derniers vers de la pièce qui a précédé immédiatement The Dreaming of the Bones dans l'œuvre de Yeats, At the Hawk's Well:

Come to me, human faces, Familiar memories; [...] Being but a mouthful of air, I am content to perish; I am but a mouthful of sweet air ${ }^{40}$. (CP, 219)

Le spectral, le fantôme c'est donc aussi le pneuma, le souffle : "plus rien, restait le souffle " dit Mallarmé sur la demeure d'Igitur. Le lyrisme tel que le travaille la modernité après Mallarmé problématise de façon nouvelle la question de la présence, très loin du bel canto romantique. De la même façon dans le Nô, la voix et la musique préludent au surgissement des fantômes qui vont prendre forme, prendre corps. Parce que voix et musique sont aussi souffle et rythme, la danse n'a pas d'autre racine que la musicalité : la maitrise du souffle est à l'origine de la matérialisation par le mouvement de l'invisible dans l'espace ${ }^{41}$. De ce fait, le drame ne repose plus sur le principe de représentation, mais au contraire inter-

39. Jacques Derrida, De la grammatologie, op. cit., p. 273.

40. William B. Yeats, Collected Plays, op. cit., p. 219.

41. On retrouve là le cour de ce qui fait l'art de l’acteur de Nô. Comme le dit Monique Borie, « La plénitude de son art se réalise dans l'accord parfait du chant et de la danse, du souffle et du mouvement, de ce qui se donne à voir et de ce qui se donne à entendre, du voir et de la voix ". Ainsi le yugen, au Japon, est cet art de l'acteur qui chante et qui danse, et qui peut le temps d'une brève incarnation être habité par l'esprit d'un mort ou d'un dieu. Voir Monique Borie, Le Fantôme ou le théâtre qui doute, Paris, Actes Sud, 1997, p. 96. 
roge la représentabilité. Son objet est le retour du disparu, par le pouvoir magique de la nomination, et son horizon est l'attente d'une résurrection épiphanique par la parole chantée. L'écriture elle-même se fait le lieu de l'affleurement du songe, par sa capacité à faire surgir les choses tout en manifestant leur absence. La scène théâtrale, dans cette pièce et peut-être dans toutes les " Pièces pour danseurs " de Yeats, n'est qu'une variation poétique sur la question : "Who's there?"

Au passage, signalons qu'en dévouant ainsi à la parole cette mission de convocation, le poète adopte une posture biblique. Dans ses commentaires sur la pièce, Yeats signale son analogie avec une des visions du prophète Ezechiel : celle, précisément, des ossements qui se réveillent et parlent (Ezechiel, 37-1-14). Dans The Dreaming of the Bones, le déploiement de la parole parlée et chantée des musiciens fait basculer le texte dans le rite, voire la liturgie : une cérémonie de parole et de chant convoque êtres et choses dans un monde spectral, et la scène n'est que le double d'une scène primitive qui revient hanter le temps d'une représentation le monde présent. Un dernier fantôme, glissé discrètement mais judicieusement au début de la pièce, prend alors toute sa valeur, lorsque l'on comprend que le poèteécrivain en est la résurgence présente :

Have not old writers said That dizzy dreams can spring From the dry bones of the dead? 